OPEN ACCESS

Edited by:

Antonio Arauz,

Instituto Nacional de Neurología y

Neurocirugía Manuel Velasco

Suárez, Mexico

Reviewed by:

Terence J. Quinn,

University of Glasgow,

United Kingdom

Aristeidis H. Katsanos,

McMaster University, Canada

${ }^{*}$ Correspondence:

Frini Karayanidis

frini.karayanidis@newcastle.edu.au

Specialty section: This article was submitted to Stroke,

a section of the journa

Frontiers in Neurology

Received: 09 January 2020 Accepted: 09 March 2020

Published: 21 April 2020

Citation:

Nicolas K, Levi C, Evans T-J, Michie PT, Magin P, Quain D, Bivard A and Karayanidis $F(2020)$ Cognition in the First Year After a Minor Stroke, Transient Ischemic Attack, or Mimic Event and the Role of Vascular Risk Factors. Front. Neurol. 11:216. doi: 10.3389/fneur.2020.00216

\section{Cognition in the First Year After a Minor Stroke, Transient Ischemic Attack, or Mimic Event and the Role of Vascular Risk Factors}

\author{
Korinne Nicolas 1,2,3, Christopher Levi ${ }^{2,3,4,5}$, Tiffany-Jane Evans ${ }^{1}$, Patricia T. Michie ${ }^{1,2}$, \\ Parker Magin 1,2,3, Debbie Quain ${ }^{1,2}$, Andrew Bivard ${ }^{3,6}$ and Frini Karayanidis ${ }^{1,2,3 *}$ \\ ${ }^{1}$ Functional Neuroimaging Laboratory, School of Psychology, University of Newcastle, Newcastle, NSW, Australia, ${ }^{2}$ Brain and \\ Mental Program, Hunter Medical Research Institute, Newcastle, NSW, Australia, ${ }^{3}$ Priority Research Centre for Stroke and \\ Brain Injury, University of Newcastle, Newcastle, NSW, Australia, ${ }^{4}$ University of New South Wales, Sydney, NSW, Australia, \\ ${ }^{5}$ Sydney Partnership for Health, Education, Research and Enterprise, Sydney, NSW, Australia, ${ }^{6}$ Melbourne Brain Center, \\ Royal Melbourne Hospital, University of Melbourne, Melbourne, VIC, Australia
}

Background: Cognitive impairment following a minor stroke or transient ischemic attack (TIA) is common; however, due to diagnostic difficulties, the prevalence and underlying cause of impairment remain poorly defined. We compared cognition in patients after a minor stroke, TIA, or mimic event at three time points in the first year following the event. We examine whether cognitive impairment occurs following these events and whether this impairment differs based on the event type. Further, we measure whether these findings persist after controlling for age, education, and the presence of vascular risk factors and whether the presence of vascular risk factors, independent of event etiology, is associated with cognitive impairment. Lastly, we investigate whether increased stroke risk, as assessed by the ABCD2, is associated with reduced cognition.

Methods: Medical information, a cognitive screening test, and a measure of executive functioning were collected from 613 patients (123 minor stroke, 175 TIA, and 315 mimics) using phone interviews at three time points in the first year following the event. Linear mixed models were used to determine the effect of event type, vascular risk factors, and predicted stroke risk on cognitive performance while controlling for confounders.

Results: There was no relationship between event type and performance on either cognitive measure. When all confounders are controlled for, performance on the cognitive screening test was uniquely accounted for by the presence of heart failure, myocardial infarction, angina, and hypertension (all $p<0.047$ ), whereas the measure of executive functioning was uniquely accounted for by the presence of hypertension and angina (all $p<0.032$ ). Increased stroke risk also predicted performance on the cognitive screening test and the measure of executive functioning (all $p<0.002$ ).

Conclusions: Our findings indicate that cognitive impairment following a minor stroke or TIA may be attributed to the high prevalence of chronic vascular risk factors in these patients. This highlights the importance of long-term management of vascular risk factors 
beyond event recovery to reduce the risk of cognitive impairment. Increased stroke risk (i.e., ABCD2 score) was also associated with reduced cognition, suggesting that it may be helpful in signaling the need for further cognitive evaluation and intervention post-event.

Keywords: cognition, transient ischemic attack, minor stroke, vascular risk factors, cognitive impairment

\section{INTRODUCTION}

Minor stroke and transient ischemic attack (TIA) are brief episodes of neurological dysfunction that lie on a spectrum of severity, with minor stroke considered a more severe neurological event (1). While clinical symptoms are transient, there is increasing evidence of persistent moderate cognitive impairment $(2,3)$. Moreover, patients frequently complain of persistent memory problems, confusion, and fatigue (4) that interfere with activities of daily living and reduce quality of life (5). The rate of moderate (29-68\%) and severe (5-22\%) cognitive impairment varies across studies (3). This variability is likely to be partly due to clinical factors that affect diagnosis, as symptoms are often resolved before clinical assessment and diagnosis is often based on clinician's interpretation of the patients' recollection of the event. Moreover, as some nonvascular conditions (e.g., episodes of migraine, seizure, or vestibular disturbance) mimic the symptoms of minor stroke and TIA (6), it is possible that some studies that do not include specialist diagnosis may inadvertently include a proportion of mimic patients for whom changes in cognitive functioning have not been specifically established.

Estimates of cognitive impairment in minor stroke and TIA patients may also be inflated by the failure to control for the pre-event presence of vascular (e.g., hypertension and hyperlipidemia) and non-vascular (e.g., age and education level) risk factors that are known to impact cognitive functioning (7). Vascular risk factors accelerate the progression of atherosclerosis, resulting in decreased blood flow and higher risk of white matter damage (8) and vascular events (9). White matter damage associated with vascular risk factors is most prominent in the prefrontal cortex, an area that supports executive functions (2, 10). Age-related changes in brain structure and cognition are accelerated 2-fold over a 5-year period in people with vascular risk factors compared to age-matched controls $(11,12)$. Minor stroke and TIA patients have a greater prevalence of vascular risk factors than do healthy controls $(2,13)$ and a greater rate of neural atrophy over a 3-year period (14). Therefore, cognitive complaints associated with these minor neurological events may, in fact, arise from more chronic vascular disease processes predating or following on from the focal ischemic damage caused by the event itself.

In this study, we investigate cognitive functioning during the first year after a minor stroke, a TIA, or a mimic event. We examine whether cognitive functioning varies as a function of event type and, if so, whether these differences can be attributed to the differential presence of vascular risk factors. As a minor stroke is a more severe vascular event than a TIA, we expect that it will be associated with poorer cognitive performance. Likewise, we expect that TIA will be associated with poorer cognition than the range of transient mild symptoms that characterize mimic events. We expect that vascular risk factors will also be associated with poorer cognitive ability, independently of event etiology. However, if the neurological impact of minor stroke and TIA is greater than the impact of vascular risk factors, event type effects on cognition are expected to persist when controlling for vascular risk factors. Finally, we examine the clinically important question of whether risk of future stroke, assessed using the ABCD2, can predict cognition, independently of event type and vascular risk factors.

\section{METHODS}

The International Systems of Care and Patient Outcomes in Minor Stroke and TIA (INSIST) is a longitudinal, communitybased inception cohort study that was approved by the Hunter New England Health and the University of Newcastle Human Research Ethics Committees (12/04/18/4.02; H-2012-0154). All patients provided written informed consent.

Six hundred and thirteen patients who presented to 16 primary and secondary care practices, including general practices, emergency departments, acute neurovascular clinics, stroke units, and hospitals in the Hunter and Manning Valley regions of New South Wales, Australia, with a possible stroke or TIA diagnosis were recruited into the study (15).

Adjudication of the presenting event as a minor stroke, TIA, or mimic event was completed by a specialist clinical panel including two stroke neurologists, a general practitioner, and an academic neuroradiologist using a standardized clinical definition across all practices (see Table 1). Adjudication was based on a consensus decision across the panel using the patient's narrative of the baseline event, clinical symptom

TABLE 1 | Criteria for adjudication of a TIA, minor stroke, or mimic event in the INSIST study.

\begin{tabular}{ll}
\hline Adjudicated group & INSIST criteria \\
\hline Transient ischemic attack & $\begin{array}{l}\text { Rapidly developing clinical signs of focal } \\
\text { disturbance of cerebral function lasting less than } \\
24 \mathrm{~h} \text { with no apparent non-vascular cause } \\
\text { Minor stroke }\end{array}$ \\
& $\begin{array}{l}\text { A stroke with a National Institutes of Health Stroke } \\
\text { Scale score of less than or equal to } 4 \text { lasting more } \\
\text { than } 24 \mathrm{~h}\end{array}$ \\
Mimic event & Neurological disturbance with no apparent vascular \\
& cause
\end{tabular}


duration, and type (i.e., positive or negative), modality (e.g., motor or sensory), and medical information (i.e., neuroimaging, clinical, and radiological information). As the study recruited from primary care facilities without access to medical imaging facilities, clinical MRI was unavailable for many patients.

At baseline, 123 events were adjudicated as a minor stroke, 175 as a TIA, and 315 as a mimic (see Supplementary Table 1 for information on neurovascular event subtype, territory, and mimic diagnosis). Recurrent events that occurred after the baseline event were clinically adjudicated at each interview using the same adjudication panel, criteria, and medical information as baseline events. However, as the focus of this study was to examine the effect of a baseline event on cognition, only the patient's baseline event diagnosis was used to determine event type for this study.

\section{Procedure}

Following their index event, participants completed three phone interviews which included demographic, neurological, cognitive, and affective measures. Due to logistical factors in recruitment and interview scheduling, the time from the baseline event to each interview varied across participants. As a result, we used time since event rather than interview occasion to assess change in outcomes post-event. Specifically, time was converted into a categorical variable based on days since the baseline event, producing three time points. Time Point 1 included interviews conducted 80 days post-event, Time Point 2 included interviews conducted 81-335 days post-event, and Time Point 3 included interviews conducted more than 335 days post-event (maximum 1,000 days; see Table 2). The majority of first, second, and third interviews were included in time points 1,2 , and 3 , respectively.

\section{Dependent Variables: Cognitive Functioning}

The Telephone Interview for Cognitive Status [TICS; (16)] is an 11-item cognitive screening test (maximum score 41). The TICS has been validated against the Mini-Mental State Examination (17).

The semantic Verbal Fluency Task [VFT; animal names in $1 \mathrm{~min},(18)]$ was used as a measure of executive functions. It has been validated for use over the telephone (19) with scores over 13 indicating healthy performance in older adults (20).

\section{Independent Variables: ABCD2 Score, Vascular Risk Factors, Patient Demographics, Mood}

The ABCD2 score is a risk stratification tool designed to identify the predicted risk of stroke in patients who present to acute care with a minor stroke or a TIA. The ABCD2 is used as a guide to emergency assessment and treatment, predicting the risk of stroke at 2, 7, and 90 days post-event (21). The ABCD2 combines significant clinical features of a minor neurological event and vascular risk factors to categorize a patient's risk of future stroke. As is common in the literature $(22,23), \mathrm{ABCD} 2$ scores were analyzed as a categorical variable with three groups, each indicating a different risk of future stroke: low (scores of $0-3$ ), moderate (scores of 4-5), and high (scores of 6-7).

Patient demographics included age at the time of event, sex, years of education, and the presence of vascular risk factors (hypertension, hyperlipidemia, myocardial infarction, heart failure, angina, peripheral vascular disease, atrial fibrillation, and diabetes). The presence of vascular risk factors at baseline was based on clinical diagnosis by the attending clinician.

The Hospital Anxiety and Depression Scale [HADS, (24)] is a self-assessment scale for depression and anxiety in a medical environment with good psychometric properties in psychiatric, primary care patients and the general population (25). A score of $>11$ (range $0-21$ ) on the HADS indicates a higher risk of depression (24).

The modified Rankin Scale [mRS, (26)] measures the degree to which physical disability following a stroke impedes daily activities. Scores range from 0 to 6 , with higher scores indicating greater levels of disability. mRS scores were obtained at each time point, and a pre-event mRS score was derived retrospectively using information from patients and carers. Pre- and post-event mRS scores show good concurrent validity and are a robust predictor of prognosis $(27,28)$.

\section{Statistics}

Event type effects (minor stroke, TIA, and mimic) were examined using a one-way ANOVA for continuous variables (age), a Kruskal-Wallis $H$ test for discrete variables (number of vascular risk factors, categorized ABCD2 score, and mRS), and a chisquare test of independence for categorical variables [attrition, vascular risk factor presence, sex, depression ( $>11$ on the HADS), and low education ( $\leq 12$ years of formal education), (29)] and

TABLE 2 | Summary of recategorization from interview occasion (columns) to time points (rows) used to analyze time since event in lieu of interview occasions.

\begin{tabular}{|c|c|c|c|c|c|}
\hline & & \multicolumn{3}{|c|}{ Interview occasion } & \\
\hline & & 1 & 2 & 3 & \\
\hline \multicolumn{2}{|c|}{ Days post-event (mean \pm SD) } & $57 \pm 50$ & $150 \pm 53$ & $381 \pm 41$ & \\
\hline Time point & Days post-event & $n$ & $n$ & $n$ & Total $N$ \\
\hline 1 & $0-80$ & 497 & 0 & 1 & 498 \\
\hline 2 & 81-335 & 113 & 603 & 7 & 723 \\
\hline 3 & $336+$ & 3 & 5 & 603 & 611 \\
\hline Total N & & 613 & 608 & 611 & 1,832 \\
\hline
\end{tabular}


summarized using the mean and standard deviation. Significant main effects of event type were examined using simple contrasts between group pairs. Post-hoc power analysis found that the INSIST sample size had $80 \%$ power to detect a moderate effect size of 0.35 (Cohen's $D$ ) for these continuous outcomes when comparing any two groups.

Linear mixed models were built in Stata (version 14) to examine crude main effects of each independent variable (IV; event type, grouped ABCD2 score, and vascular risk factors) on each dependent variable (DV; TICS and VFT) across time points. The crude model for each IV also included a term for time point as a categorical variable and a random effect to control for the correlation of repeated measures on individuals. Adjusted models were created to examine whether any significant main effect of the IV survived after adjusting for potential confounders for each IV-DV relationship.

Directed acyclic graphs (DAGs) for each model were derived to theoretically characterize the relationship between each IV and DV and to identify confounders that needed to be controlled in order to estimate the total effect of the IV on the DV (30). Based on the DAG, to identify the independent effect of event type on cognition (Model 1), we needed to control for age and all vascular risk factors. For each vascular risk model (Model 2), we needed to control for education, depression, age, and all other vascular risk factors. For these models, the DAG identified that event type was a potential mediator. To examine whether event type affected the relationship between vascular risk factors and cognition, in initial analyses, event type was included in each vascular risk model. As its inclusion scarcely changed findings, event type was not included in the vascular risk models presented below. For the ABCD2 model (Model 3), we controlled for event type and all vascular risk factors excluding hypertension and diabetes, as they contribute to the ABCD2 score. For all models, there was no evidence of violation of the model assumptions of linearity, non-constant variance, and normality of the residuals.

\section{RESULTS}

Of the 613 participants, two were missing both TICS and VFT scores and were removed from further analyses $(N=611)$. We used mixed model analysis for these longitudinal data, so data from all 611 participants contributed to the analyses, even if there were missing data on one or two time points. Table 3 summarizes the number of participants who completed the TICS and VFT at each interview occasion.

Twenty participants completed the TICS and VFT only on their first interview occasion and were thereafter lost to followup. These 20 participants were older $(p<0.001)$ and performed more poorly on both the TICS and VFT measures at Time Point 1 ( $p=0.001$ and $p=0.036$, respectively; data not tabulated) than participants who contributed to additional data points. Excluding these participants from all analyses below did not change the pattern of findings, so they were retained.

As shown in Table 4, the three event types differed significantly on a number of demographic and risk variables. There were significant group differences in sex $(p<0.001)$,
TABLE 3 | Summary of the number of participants who provided cognitive measures at each interview.

\begin{tabular}{lcc}
\hline Number of interview(s) completed & TICS & VFT \\
\hline Interview 1 Only & $20(3.3 \%)$ & $20(3.3 \%)$ \\
Interview 2 Only & $1(0.2 \%)$ & $3(0.5 \%)$ \\
Interview 3 Only & 0 & $1(0.2 \%)$ \\
Interviews 1 and 2 & $25(4.1 \%)$ & $27(4.4 \%)$ \\
Interviews 1 and 3 & $12(2.0 \%)$ & $15(2.5 \%)$ \\
Interviews 2 and 3 & $1(0.2 \%)$ & $14(2.3 \%)$ \\
All interviews & $552(90.3 \%)$ & $531(86.9 \%)$ \\
\hline Total data obtained & 611 & 611 \\
\hline
\end{tabular}

ABCD2 categories $(p<0.001)$, number of vascular risk factors $(p<0.001)$, and prevalence of all vascular risk factors excluding hyperlipidemia and diabetes (all $p<0.033$ ). Comparisons (data not tabulated) revealed that minor stroke and TIA patients had a higher percentage of males compared with mimic patients $(p$ $<0.001$ ). Minor stroke patients had significantly higher ABCD2 scores and number of vascular risk factors compared to both TIA and mimic patients, and TIA patients had higher ABCD2 scores and number of vascular risk factors compared to mimic patients (all $p<0.001$ ). Group differences regarding the presence of vascular risk factors were driven by a greater prevalence in minor stroke and TIA patient groups (see Table 4).

\section{Cognitive and Disability Changes Across Time Points}

As shown in Table 5, TICS scores increased progressively over post-event time points $\left[N=611, \chi^{2}(2)=74.17, p<0.001\right.$; Time Points 1 to 2 : $95 \% \mathrm{CI},-1.11$ to -0.28 ; Time Points 2 to $3: 95 \% \mathrm{CI}$, 0.02 to 0.83 ]. Although statistically significant, the total increase of 1.1 score (maximum $=41$ ) is not clinically significant. VFT scores did not vary across time points $\left[N=611, \chi^{2}(2)=0.65\right.$, $p=0.724]$. mRS scores varied significantly over time $[N=611$, $\left.\chi^{2}(3)=92.67, p<0.001\right]$. There was a substantial increase in disability from pre-event to Time Point 1 (95\% CI, 0.11 to 0.21 ) and a smaller but progressive increase thereafter (Time Points 1 to 2: $95 \%$ CI, 0.12 to 0.22 ; Time Points 2 to $3: 95 \% \mathrm{CI}, 0.18$ to $0.28)$. Depression scores varied significantly over time $[N=611$, $\left.\chi^{2}(2)=32.71, p<0.001\right]$, decreasing slightly from Time Points 1 to $2(95 \% \mathrm{CI},-0.49$ to 0.17$)$ and significantly from Time Points 2 to 3 (95\% CI, -0.94 to -0.25$)$.

Time point was included in all three models below but did not interact with either TICS or VFT in any of the three models and will not be discussed further (all $p>0.150$; data not tabulated).

\section{Model 1: Event Type on Cognition}

In the crude main effects model for event type (Table 6), TICS scores did not vary with event type. There was a small but significant effect of event type on VFT, with simple comparisons indicating that minor stroke patients performed more poorly than mimic patients ( $p=0.008$; data not tabulated). Including age as a potential confounder (adjusted model) reduced all 
TABLE 4 | All participants' sociodemographic, vascular, and lifestyle risk factors by event type.

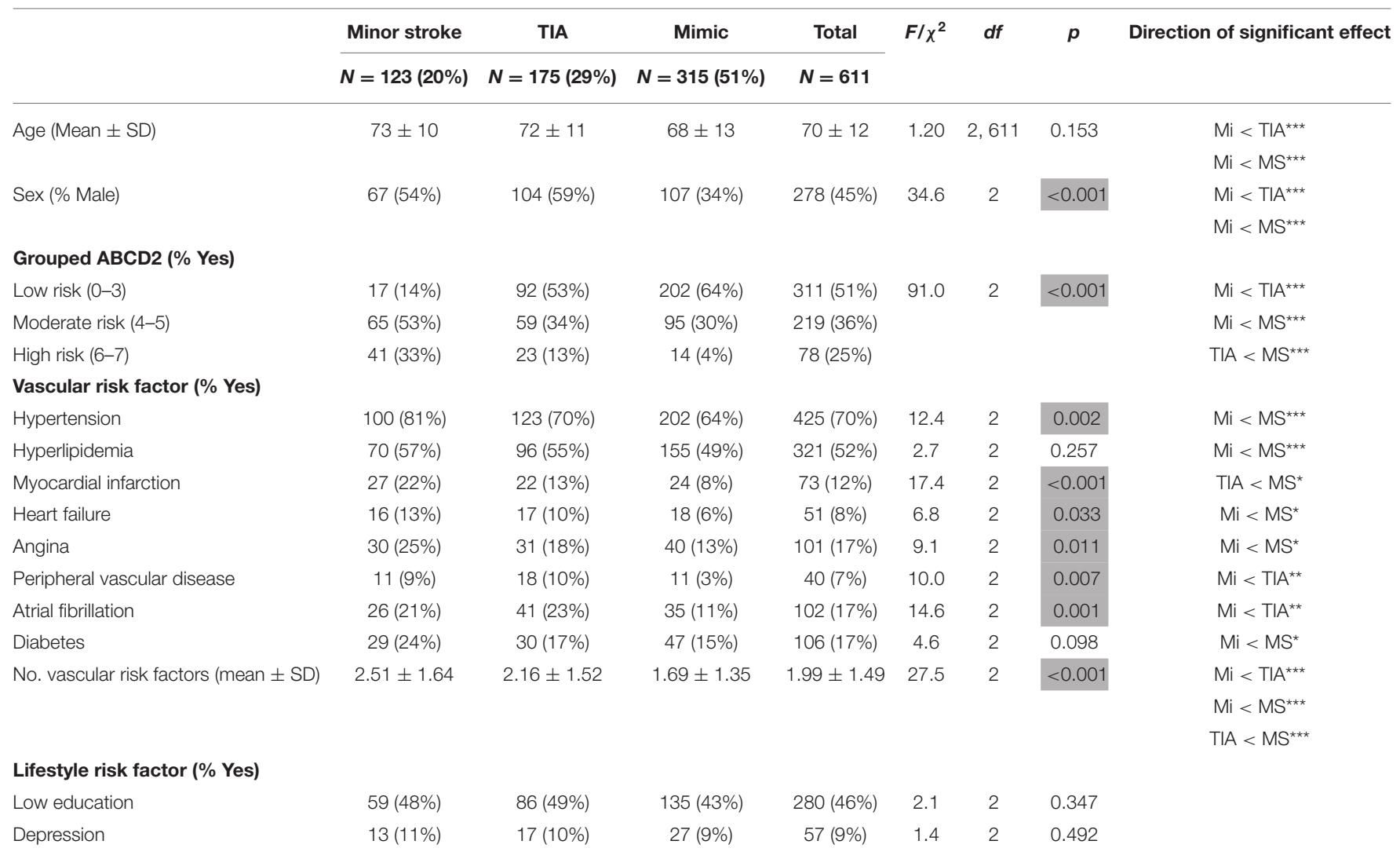

${ }^{*} p<0.05,{ }^{* *} p<0.010,{ }^{* \star *} p<0.001$, Mi, mimic; MS, minor stroke. Shaded $p$-values indicate $p<0.05$.

TABLE 5 | Mean cognitive and disability measures over time points.

\begin{tabular}{|c|c|c|c|c|c|c|}
\hline & Minor stroke & TIA & Mimic & $\chi^{2}$ & $d f$ & $p$ \\
\hline \multicolumn{7}{|c|}{ Telephone interview for cognitive status (Mean \pm SD) } \\
\hline Time 1 & $32.85 \pm 3.83$ & $33.47 \pm 3.83$ & $33.82 \pm 3.53$ & 74.2 & 2 & $<0.001$ \\
\hline Time 2 & $33.76 \pm 3.65$ & $34.53 \pm 3.48$ & $34.21 \pm 3.45$ & & & \\
\hline Time 3 & $34.59 \pm 4.01$ & $34.53 \pm 3.56$ & $34.72 \pm 3.74$ & & & \\
\hline Mean over time & $33.73 \pm 3.83$ & $34.18 \pm 3.62$ & $34.25 \pm 3.57$ & & & \\
\hline Time 2 & $18.86 \pm 6.45$ & $19.27 \pm 6.45$ & $19.60 \pm 6.28$ & & & \\
\hline Time 3 & $18.75 \pm 6.39$ & $18.72 \pm 6.42$ & $19.88 \pm 6.40$ & & & \\
\hline Mean over time & $18.42 \pm 6.16$ & $18.92 \pm 6.16$ & $19.90 \pm 6.28$ & & & \\
\hline \multicolumn{7}{|c|}{ Modified rankin scale (Mean \pm SD) } \\
\hline Pre-event & $0.72 \pm 1.13$ & $0.75 \pm 1.08$ & $0.68 \pm 1.02$ & 92.7 & 2 & $<0.001$ \\
\hline Mean over time & $1.15 \pm 1.14$ & $0.82 \pm 1.10$ & $0.75 \pm 1.04$ & & & \\
\hline \multicolumn{7}{|c|}{ Hospital anxiety and depression scale (Depression component) (Mean \pm SD) } \\
\hline Time 1 & $3.31 \pm 0.19$ & $2.91 \pm 0.17$ & $2.94 \pm 0.15$ & 32.7 & 2 & $<0.001$ \\
\hline Time 2 & $3.16 \pm 0.18$ & $2.75 \pm 0.15$ & $2.78 \pm 0.13$ & & & \\
\hline Time 3 & $2.72 \pm 0.18$ & $2.31 \pm 0.17$ & $2.34 \pm 0.14$ & & & \\
\hline Mean over time & $3.06 \pm 0.18$ & $2.66 \pm 0.16$ & $2.69 \pm 0.14$ & & & \\
\hline
\end{tabular}

Shaded $p$-values indicate $p<0.05$. 
TABLE 6 | Effects of event type on TICS and VFT.

\begin{tabular}{|c|c|c|c|c|c|c|c|c|c|}
\hline \multicolumn{10}{|c|}{ Cognitive measures by event type } \\
\hline \multirow[b]{2}{*}{ Outcome } & \multicolumn{3}{|c|}{ Crude } & \multicolumn{3}{|c|}{ Adjusted } & \multicolumn{3}{|c|}{ Final } \\
\hline & $\beta$ & $95 \% \mathrm{Cl}$ & $p$ & $\beta$ & $95 \% \mathrm{Cl}$ & $p$ & $\beta$ & $95 \% \mathrm{Cl}$ & $p$ \\
\hline \multicolumn{10}{|c|}{ Telephone interview for cognitive status } \\
\hline MS-TIA & -0.60 & -1.4 to 0.2 & 0.213 & -0.56 & -1.3 to 0.2 & 0.252 & -0.32 & -1.2 to 0.1 & 0.191 \\
\hline Mimic-TIA & 0.00 & -0.6 to 0.6 & & -0.41 & -1.0 to 0.2 & & -0.53 & -2.3 to 0.7 & \\
\hline MS-Mimic & -0.60 & -1.2 to 0.1 & & -0.15 & -0.8 to 0.5 & & 0.21 & -0.4 to 0.8 & \\
\hline \multicolumn{10}{|c|}{ Verbal fluency test } \\
\hline MS-TIA & -0.69 & -2.0 to 0.6 & 0.022 & -0.60 & -1.8 to 0.6 & 0.441 & -0.24 & -1.4 to 0.9 & 0.910 \\
\hline Mimic-TIA & 0.87 & -0.1 to 1.9 & & 0.10 & -0.9 to 1.1 & & -0.04 & -1.0 to 0.9 & \\
\hline MS-Mimic & -1.60 & -2.7 to -0.4 & & -0.70 & -1.8 to 0.4 & & -0.20 & -1.3 to 0.9 & \\
\hline
\end{tabular}

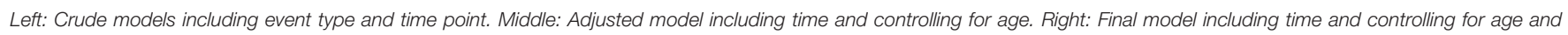
vascular risk factors. Shaded beta values indicate significant confidence intervals and significant findings $(\alpha<0.05)$.

coefficients and removed the relationship between VFT scores and event type. The final TICS and VFT models that controlled for both age and vascular risk factors resulted in further reduced coefficients. The addition of depression in the model scarcely affected coefficients from those presented and was not included in any of the event type models.

\section{Model 2: Vascular Risk Factors on Cognition}

Table 7 shows that both TICS and VFT scores were significantly lower in the presence of most individual vascular risk factors. For TICS scores, the strongest predictor was heart failure, followed in decreasing order by myocardial infarction, angina, peripheral vascular disease, hypertension, and atrial fibrillation. For VFT, the strongest predictor was again heart failure, followed by hypertension, angina, myocardial infarction, peripheral vascular disease, atrial fibrillation, and hyperlipidemia. Controlling for age and education reduced the strength of the effect of each vascular risk factor on both TICS and VFT. The final models for each vascular risk factor also controlled for all other vascular risk factors. As shown in Table 7, when controlling for other vascular risk factors, the only vascular risk factors to uniquely significantly predict TICS were, in descending order, heart failure, myocardial infarction, angina, and hypertension. For VFT scores, only hypertension and angina remained as unique predictors. The addition of depression and event type scarcely altered coefficients from those presented above and therefore were not included in any of the vascular risk factor models.

\section{Model 3: ABCD2 on Cognition}

Table 8 shows that greater risk of stroke was associated with significantly lower TICS and VFT scores. All relationships remained significant when controlling solely for event type (adjusted model) and together with vascular risk factors (final model). Simple comparisons (data not tabulated) revealed significant differences on TICS scores between all groups (low risk vs. moderate risk $p=0.008$, low vs. high $p<0.001$, and moderate vs. high $p=0.036)$ and on VFT scores between low- and moderate-risk groups $(p=0.002)$ and low- and highrisk groups $(p=0.009)$. Performance on the VFT did not differ between moderate- and high-risk groups $(p=0.572)$. The addition of depression in the model scarcely affected coefficients from those presented and was not included in any of the ABCD2 models.

\section{DISCUSSION}

The present study used a cognitive screening test (TICS) and a brief measure of executive functioning (VFT) to investigate differences in the level and progression of cognitive ability in the first year after a minor stroke, TIA, or mimic event. We sought to identify whether cognitive ability was differentially impacted by event severity, independently of the presence of coexisting, chronic vascular disease processes associated with vascular risk factors.

Across the first year following a minor neurological event, there was a small but statistically significant improvement in performance on the cognitive screening test but not the measure of executive functioning. The increase in TICS score was not clinically significant; did not differ by event type, presence of vascular risk factor, or risk of future stroke; and remained when removing the few participants (3\%) who only completed the first interview. This small increase in TICS may reflect slight recovery of cognition following a brief disruption after an event and/or test practice effect.

\section{Event Type}

Event type had a significant effect on the measure of executive functioning that was driven by poorer performance in the minor stroke group compared to the mimic group. This effect was weak and was removed after controlling for either vascular risk factors or age. In conclusion, in this large sample and using broad cognitive screening tasks, differences in cognitive functioning between minor stroke, TIA, and mimic events in the first year post-event were weak and attributable to differential prevalence of vascular risk factors and/or age. Alternatively, it 
TABLE 7 | Effects of vascular risk factors on TICS and VFT.

\begin{tabular}{|c|c|c|c|c|c|c|c|c|c|}
\hline \multicolumn{10}{|c|}{ Cognitive measures by vascular risk factors } \\
\hline \multirow[b]{2}{*}{ Outcome } & \multicolumn{3}{|c|}{ Crude } & \multicolumn{3}{|c|}{ Adjusted } & \multicolumn{3}{|c|}{ Final } \\
\hline & $\beta$ & $95 \% \mathrm{Cl}$ & $p$ & $\beta$ & $95 \% \mathrm{Cl}$ & $p$ & $\beta$ & $95 \% \mathrm{Cl}$ & $p$ \\
\hline \multicolumn{10}{|c|}{ Telephone interview for cognitive status } \\
\hline Hypertension & -1.43 & -2.0 to -0.9 & $<0.001$ & -0.74 & -1.3 to -0.2 & 0.009 & -0.68 & -1.3 to -0.1 & 0.020 \\
\hline Hyperlipidemia & -0.36 & -0.9 to 0.2 & 0.183 & -0.12 & -0.6 to 0.4 & 0.633 & 0.28 & -0.2 to 0.8 & 0.296 \\
\hline Myocardial infarction & -1.93 & -2.7 to -1.1 & $<0.001$ & -1.36 & -2.1 to -0.6 & $<0.001$ & -0.88 & -1.7 to -0.1 & 0.004 \\
\hline Heart failure & -2.48 & -3.4 to -1.5 & $<0.001$ & -1.5 & -2.4 to -0.6 & 0.001 & -0.96 & -1.9 to 0.0 & 0.047 \\
\hline Angina & -1.84 & -2.5 to -1.1 & $<0.001$ & -1.21 & -1.9 to -0.5 & $<0.001$ & -0.77 & -1.5 to 0.0 & 0.038 \\
\hline Peripheral vascular disease & -1.77 & -2.8 to -0.7 & 0.001 & -0.8 & -1.8 to 0.2 & 0.119 & 0.01 & -1.0 to 1.1 & 0.983 \\
\hline Atrial fibrillation & -1.13 & -1.8 to -0.4 & 0.002 & -0.3 & -1.0 to 0.4 & 0.394 & -0.08 & -0.8 to 0.6 & 0.804 \\
\hline Diabetes & -0.42 & $-1-1$ to 0.3 & 0.245 & -0.33 & -1.0 to 0.3 & 0.307 & -0.08 & -0.7 to 0.6 & 0.804 \\
\hline \multicolumn{10}{|l|}{ Verbal fluency test } \\
\hline Hypertension & -3.01 & -3.9 to -2.1 & $<0.001$ & -1.69 & -2.6 to -0.8 & $<0.001$ & -1.39 & -2.3 to -0.5 & 0.004 \\
\hline Hyperlipidemia & -1.52 & -2.4 to -0.6 & $<0.001$ & -0.98 & -1.8 to -0.2 & 0.017 & -0.39 & -1.2 to 0.5 & 0.368 \\
\hline Myocardial infarction & -2.5 & -3.9 to -1.1 & $<0.001$ & -1.43 & -2.7 to -0.2 & 0.024 & -0.53 & -1.9 to 0.8 & 0.436 \\
\hline Heart failure & -3.98 & -5.6 to -2.4 & $<0.001$ & -2.25 & -3.7 to -0.8 & 0.003 & -1.46 & -3.0 to 0.1 & 0.067 \\
\hline Angina & -2.90 & -4.1 to -1.7 & $<0.001$ & -1.77 & -2.9 to -0.7 & 0.001 & -1.31 & -2.5 to -0.1 & 0.032 \\
\hline Peripheral vascular disease & -2.21 & -4.0 to -0.4 & 0.016 & -0.55 & -2.2 to 1.1 & 0.516 & 0.74 & -1.1 to 2.5 & 0.404 \\
\hline Atrial fibrillation & -2.04 & -3.2 to -0.9 & $<0.001$ & -0.53 & -1.6 to 0.6 & 0.348 & -0.34 & -1.4 to 0.8 & 0.555 \\
\hline Diabetes & -0.86 & -2.0 to 0.3 & 0.146 & -0.33 & -1.7 to 0.4 & 0.219 & -0.14 & -1.2 to 0.9 & 0.798 \\
\hline
\end{tabular}

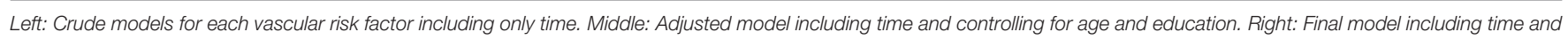
controlling for age, education, and all other vascular risk factors. Shaded beta values indicate significant confidence intervals and significant findings ( $\alpha<0.05)$.

TABLE 8 | Effects of ABCD2 on cognition.

Cognitive Measures by ABCD2

\begin{tabular}{|c|c|c|c|c|c|c|c|c|c|}
\hline \multirow[b]{2}{*}{ Outcome } & \multicolumn{3}{|c|}{ Crude } & \multicolumn{3}{|c|}{ Adjusted } & \multicolumn{3}{|c|}{ Final } \\
\hline & $\beta$ & $95 \% \mathrm{Cl}$ & $p$ & $\beta$ & $95 \% \mathrm{Cl}$ & $p$ & $\beta$ & $95 \% \mathrm{Cl}$ & $p$ \\
\hline \multicolumn{10}{|c|}{ Telephone interview for cognitive status } \\
\hline Mod-Low & -0.82 & -1.4 to -0.3 & $<0.001$ & -0.85 & -1.5 to -0.2 & $<0.001$ & -0.79 & -1.37 to -0.2 & $<0.001$ \\
\hline High-Low & -1.77 & -2.5 to -0.9 & & -1.82 & -2.7 to -0.9 & & -1.7 & -2.57 to -0.8 & \\
\hline High-Mod & -0.94 & -1.8 to -0.8 & & -0.97 & -1.9 to -0.1 & & -0.91 & -1.8 to -0.1 & \\
\hline \multicolumn{10}{|c|}{ Verbal fluency test } \\
\hline Mod-Low & -1.78 & -2.7 to -0.8 & $<0.001$ & -1.6 & -2.7 to -0.7 & $<0.001$ & -1.53 & -2.5 to -0.6 & 0.002 \\
\hline High-Low & -2.58 & -3.9 to -1.2 & & -2.3 & -3.8 to -0.8 & & -1.94 & -3.4 to -0.5 & \\
\hline High-Mod & -0.80 & -2.2 to 0.6 & & -0.66 & -2.1 to 0.8 & & -0.41 & -1.8 to 1.0 & \\
\hline
\end{tabular}

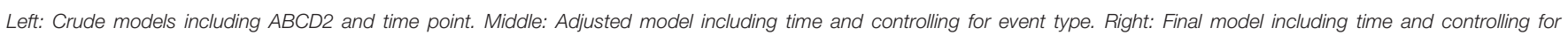

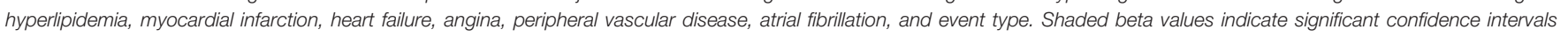
and significant findings $(\alpha<0.05)$.

is possible that the broad cognitive measures used in this study were not sufficiently sensitive to detect subtle differences as a function of event type. It is possible that more sensitive cognitive tasks and functional brain imaging measures may reveal more subtle impairments.

Other studies suggest that both neural structure and cognitive function are affected in the subacute stage following a minor stroke or TIA. Bivard et al. (31) found that, compared to healthy controls with a similar number of vascular risk factors, both minor stroke and TIA patients who had evidence of acute ischemia in brain perfusion imaging showed cerebral gray matter atrophy from baseline to 90 days post-event as well as poorer performance on executive function and attention subscales of the Montreal Cognitive Assessment (MoCA). There is less consistent evidence for long-term cognitive impairment following a TIA or minor stroke. Munir et al. (14) reported that, although minor stroke and TIA patients had a higher rate of neural atrophy and slower processing speed compared to healthy controls over a 3-year period, neither memory nor executive functions were affected. Similarly, Sachdev et al. (32) 
found that, at 3 years post-event, minor stroke and TIA patients showed reduced verbal memory but no difference in any other cognitive domain, when compared to controls. Taken together, these findings suggest that although minor stroke and TIA may be associated with cognitive decline in the early post-event periods [e.g., 90 days in Bivard et al. (31)], the effects on cognition are only transient.

\section{Vascular Risk Factors}

Consistent with many previous studies (33-37), the presence of vascular risk factors was associated with reduced performance on both the cognitive screening task and the executive functioning measure. When we examined the unique contribution of each vascular risk factor and controlled for age and education, performance on the cognitive screening test was significantly impaired, in decreasing order of impact, by the presence of heart failure, myocardial infarction, angina, and hypertension, whereas executive function scores were significantly impacted by hypertension and angina.

Minor stroke patients had a greater prevalence of each of these vascular risk factors that independently affected cognitive performance; however, inclusion of event type as a confounder did not impact the relationship between vascular risk factors and cognition. While it is well established that vascular risk factors affect cognition, this finding provides evidence that the reported cognitive dysfunction after a minor stroke or TIA may be attributed to the increased prevalence of vascular risk factors in such patients. Moreover, the robust findings between vascular risk factors and cognitive ability after controlling for a number of confounders suggest that these broad cognitive measures are sufficiently sensitive to progressive effects arising from vascular factors. This suggests that the null finding of event type (discussed above) is unlikely to be due to test insensitivity.

\section{Predicted Stroke Risk}

As expected, $\mathrm{ABCD} 2$ scores were highest in minor stroke patients, followed by TIA and mimic patients. This finding supports the use of the $\mathrm{ABCD} 2$ score as a tool to assist in diagnostic decisions, especially in clinical settings that may not have access to imaging or specialist resources. Further, we found that increased predicted risk of future stroke was associated with reduced performance on both cognitive tests, even after controlling for event type and vascular risk factors (excluding hypertension and diabetes which contribute to the ABCD2 score). As there was no evidence of a relationship between cognition and either event type or diabetes, it is likely that the relationship between cognition and stroke risk is driven by the other variables that contribute to the $\mathrm{ABCD} 2$ score, such as age and hypertension.

\section{CONCLUSION}

A large community-dwelling cohort of patients with a clinical diagnosis of minor stroke, TIA, or mimic event was assessed over the first year post-event. Level of physical disability and risk of future stroke progressively increased with event severity. However, event type was not associated with differential level of functioning on a cognitive screening test and produced only a small effect on a measure of executive functioning that was eliminated after controlling for vascular risk factors and age. In contrast, the presence of vascular risk factors and most consistently hypertension and angina, as well as predicted stroke risk, were associated with reduced performance on both the cognitive screening and the executive function measures, irrespective of event etiology and other confounders.

The absence of an effect of neurological event type on cognition, in the presence of significant effects of future stroke risk and vascular risk factors, suggests that vascular risk factors may account, at least partially, for reported levels of cognitive decline after a minor stroke or TIA. This work supports the notion that these mild neurological events have only transient effects on cognitive functioning, suggesting that the common cognitive complaints reported following such transient events may arise from the increased prevalence of chronic vascular risk factors that are present in these patients before the neurological event. This highlights the need to monitor cognitive functioning associated with vascular risk factors and, in the absence of pre-event measures, obtain estimates of pre-event cognition at the time of the presenting event (38). Finally, the ABCD2, which also loads heavily on vascular risk factors, varied across neurological event type and was associated with cognitive decline, independently of event type. This is consistent with the role of vascular risk factors in cognitive decline. Together, these findings suggest that the presence of vascular risk factors could signal the need to monitor patients' cognitive functioning trajectory and consider appropriate interventions which may aid in reducing the risk of cognitive decline by improving cognitive ability. There is emerging evidence suggesting that cognitive ability can be improved by treating vascular risk factors; for example, a review by Elias et al. (39) reports multiple studies displaying an improvement in cognitive ability after treatment with candesartan in hypertensive patients. Moreover, a review by Hajduk et al. [(40), see also (41)] reports that patients with heart failure undergoing interventions to increase cardiac function displayed cognitive improvements.

A particular strength of this study is the inclusion of a large community-dwelling cohort that consisted of patients managed entirely across a range of primary care settings as well as patients in secondary care. Event adjudication was completed by a specialist panel using general practitioner, hospital, emergency department, and specialist notes to accurately diagnose vascular risk factors. Cognitive ability was assessed using trajectory analysis at three time points after the event. Based on these findings, we conclude that clinically adjudicated mild neurological events do not significantly impact cognition in the first year post-event, whereas the presence of vascular risk factors and future stroke risk are associated with lower cognition but not differential decline over a 1-year period.

This conclusion needs to be considered in light of potential caveats that may affect its generalization. Firstly, we compared minor stroke and TIA groups against a mimic group instead of a healthy control group. While mimic patients constitute a good clinical control group, these patients had a broad range of clinical presentations and are likely to perform more 
poorly than a healthy control group. Secondly, in the absence of clinical imaging, it was not possible to use imaging-based categorization of minor stroke and TIA or to examine whether the location of an ischemic event differentially affected cognitive profile. Thirdly, we did not control for differential effects of clinical treatment or pre-/post-event medication history. Fourthly, we did not complete a comprehensive and in-depth neuropsychological assessment, and therefore, subtle cognitive differences may have been missed. Finally, in the absence of a pre-event measure of cognitive ability, it was not possible to differentiate between premorbid cognitive level and event-related cognitive effects.

Overall, these findings are consistent with other studies showing little evidence of persistent cognitive decline post-TIA or minor stroke $(14,32)$. Future work is needed to examine whether behavioral and neuroimaging measures of executive function processes and associated brain networks are more sensitive to emerging and potentially reversible levels of cognitive decline associated with these minor neurological events.

\section{DATA AVAILABILITY STATEMENT}

The datasets generated for this study are available on request to the corresponding author.

\section{ETHICS STATEMENT}

The studies involving human participants were reviewed and approved by Hunter New England Health and the University of Newcastle Human Research Ethics Committees (12/04/18/4.02;

\section{REFERENCES}

1. Ortiz GA, Sacco RL. National Institutes of Health Stroke Scale (NIHSS). Wiley StatsRef: Statistics Reference Online (2014). doi: 10.1002/9781118445112.stat06823

2. Sachdev PS, Brodaty H, Valenzuela MJ, Lorentz LJ, Looi CL, Wen $\mathrm{W}$, et al. The neuropsychological profile of vascular cognitive impairment in stroke and TIA patients. Neurology. (2004) 62:912-9. doi: 10.1212/01.wnl.0000115108.65264.4b

3. van Rooij FG, Kessels RP, Richard E, De Leeuw FE, van Dijk EJ. Cognitive impairment in transient ischemic attack patients: a systematic review. Cerebrovasc Dis. (2016) 42:1-9. doi: 10.1159/000444282

4. Turner GM, Calvert M, Feltham MG, Ryan R, Marshall T. Ongoing impairments following transient ischaemic attack: retrospective cohort study. Eur J Neurol. (2016) 23:1642-50. doi: 10.1111/ene.13088

5. Moran GM, Fletcher B, Feltham MG, Calvert M, Sackley C, Marshall T. Fatigue, psychological and cognitive impairment following transient ischaemic attack and minor stroke: a systematic review. Eur J Neurol. (2014) 21:1258-67. doi: 10.1111/ene.12469

6. Nadarajan V, Perry RJ, Johnson J, Werring DJ. Transient ischaemic attacks: mimics and chameleons. Pract Neurol. (2014) 14:23-31. doi: 10.1136/practneurol-2013-000782

7. Singer J, Trollor JN, Baune BT, Sachdev PS, Smith E. Arterial stiffness, the brain and cognition: a systematic review. Ageing Res Rev. (2014) 15:16-27. doi: 10.1016/j.arr.2014.02.002

8. Jolly TA, Bateman GA, Levi CR, Parsons MW, Michie PT, Karayanidis F. Early detection of microstructural white matter changes associated with arterial pulsatility. Front Hum Neurosci. (2013) 7:782. doi: 10.3389/fnhum.2013.00782
H-2012-0154). The patients/participants provided their written informed consent to participate in this study.

\section{AUTHOR CONTRIBUTIONS}

KN coauthored the manuscript and drafted tables and figures. CL and PM conceived the design of the study and edited drafts. T-JE supervised data analysis. PTM and AB contributed to revisions of drafts. DQ was involved in data acquisition and management. FK is the lead author, supervisor of KN's thesis and cognitive analyses, and coauthored the manuscript.

\section{FUNDING}

The InSiST study was funded by the National Health and Medical Research Council, Project APP1027794. KN was supported by a UNRSC 50:50 scholarship by the University of Newcastle, Australia and the CRE-Stroke Recovery and Rehab.

\section{ACKNOWLEDGMENTS}

The authors acknowledge the practice nurses, neurologists, and General Practitioners for their contributions to TIA/minor stroke identification and patient recruitment.

\section{SUPPLEMENTARY MATERIAL}

The Supplementary Material for this article can be found online at: https://www.frontiersin.org/articles/10.3389/fneur. 2020.00216/full\#supplementary-material
9. Wilterdink JL, Easton JD. Vascular event rates in patients with atherosclerotic cerebrovascular disease. Arch Neurol. (1992) 49:857-63. doi: 10.1001/archneur.1992.00530320089016

10. Barrick TR, Charlton RA, Clark CA, Markus HS. White matter structural decline in normal ageing: a prospective longitudinal study using tract-based spatial statistics. Neuroimage. (2010) 51:565-77. doi: 10.1016/j.neuroimage.2010.02.033

11. Debette S, Markus HS. The clinical importance of white matter hyperintensities on brain magnetic resonance imaging: systematic review and meta-analysis. BMJ. (2010) 341:c3666. doi: 10.1136/bmj.c3666

12. Kennedy KM, Raz N. Pattern of normal age-related regional differences in white matter microstructure is modified by vascular risk. Brain Res. (2009) 1297:41-56. doi: 10.1016/j.brainres.2009.08.058

13. Clarey J, Lasserson D, Levi C, Parsons M, Dewey H, Barber PA, et al. Absolute cardiovascular risk and GP decision making in TIA and minor stroke. Fam Pract. (2014) 31:664-9. doi: 10.1093/fampra/cmu054

14. Munir M, Ursenbach J, Reid M, Gupta Sah R, Wang M, Sitaram A., et al. Longitudinal brain atrophy rates in transient ischemic attack and minor ischemic stroke patients and cognitive profiles. Front Neurol. (2019) 10:18. doi: 10.3389/fneur.2019.00018

15. Levi CR, Lasserson D, Quain D, Valderas J, Dewey HM, Alan Barber P, et al. The International comparison of Systems of care and patient outcomes In minor Stroke and Tia (InSIST) study: a community-based cohort study. Int J Stroke. (2019) 14:186-90. doi: 10.1177/1747493018799983

16. Brandt J, Spencer M, Folstein M. The telephone interview for cognitive status. Neuropsychiatry Neuropsychol Behav Neurol. (1988) 1:111-7.

17. Castanho TC, Amorim L, Zihl J, Palha JA, Sousa N, Santos NC. Telephonebased screening tools for mild cognitive impairment and dementia in aging 
studies: a review of validated instruments. Front Aging Neurosci. (2014) 6:16. doi: 10.3389/fnagi.2014.00016

18. Thurstone LL. Primary Mental Abilities. Chicago, IL: University of Chicago Press (1938).

19. Marceaux JC, Prosje MA, McClure LA, Kana B, Crowe M, Kissela B, et al. Verbal fluency in a national sample: telephone administration methods. Int J Geriatr Psychiatry. (2019) 34:578-87. doi: 10.1002/gps.5054

20. Tombaugh TN, Kozak J, Rees L. Normative data stratified by age and education for two measures of verbal fluency: FAS and animal naming. Arch Clin Neuropsychol. (1999) 14:167-77. doi: 10.1016/S0887-6177(97)00095-4

21. Johnston SC, Rothwell PM, Nguyen-Huynh MN, Giles MF, Elkins JS, Bernstein AL, et al. Validation and refinement of scores to predict very early stroke risk after transient ischaemic attack. Lancet. (2007) 369:283-92. doi: 10.1016/S0140-6736(07)60150-0

22. Fothergill A, Christianson TJH, Brown RD, Rabinstein AA. Validation and refinement of the ABCD2 score: a population-based analysis. Stroke. (2009) 40:2669-73. doi: 10.1161/strokeaha.109.553446

23. Ozpolat C, Denizbasi A, Akoglu H, Onur O, Eroglu SE, Demir H. Use of $\mathrm{ABCD} 2$ risk scoring system to determine the short-term stroke risk in patients presenting to emergency department with transient ischaemic attack. $J$ Pak Med Assoc. (2013) 63:1142-6.

24. Zigmond AS, Snaith RP. The hospital anxiety and depression scale. Acta Psychiatr Scand. (1983) 67:361-70.

25. Bjelland I, Dahl AA, Haug TT, Neckelmann D. The validity of the Hospital Anxiety and Depression Scale: an updated literature review. J Psychosom Res. (2002) 52:69-77. doi: 10.1016/S0022-3999(01)00296-3

26. Rankin J. Cerebral vascular accidents in patients over the age of 60: II. Prog Scottish Med J. (1957) 2:200-15. doi: 10.1177/003693305700200504

27. Banks JL, Marotta CA. Outcomes validity and reliability of the modified rankin scale: implications for stroke clinical trials. Stroke. (2007) 38:1091-6. doi: 10.1161/01.str.0000258355.23810.c6

28. Quinn TJ, Taylor-Rowan M, Coyte A, Clark AB, Musgrave SD, Metcalf AK, et al. Pre-stroke modified rankin scale: evaluation of validity, prognostic accuracy, and association with treatment. Front Neurol. (2017) 8:275. doi: 10.3389/fneur.2017.00275

29. Nasreddine ZS, Phillips NA, Bédirian VR, Charbonneau S, Whitehead V, Collin I, et al. The Montreal Cognitive Assessment, MoCA: a brief screening tool for mild cognitive impairment. J Am Geriatr Soc. (2005) 53:695-9. doi: 10.1111/j.1532-5415.2005.53221.x

30. Thoemmes F, Rosseel Y, Textor J. Local fit evaluation of structural equation models using graphical criteria. Psychol Methods. (2018) 23:27-41. doi: $10.1037 /$ met0000147

31. Bivard A, Lillicrap T, Maréchal B, Garcia-Esperon C, Holliday E, Krishnamurthy $\mathrm{V}$, et al. Transient ischemic attack results in delayed brain atrophy and cognitive decline. Stroke. (2018) 49:384-90. doi: 10.1161/strokeaha.117.019276
32. Sachdev PS, Lipnicki DM, Crawford JD, Wen W, Brodaty H. Progression of cognitive impairment in stroke/TIA patients over 3 years. J Neurol Neurosurg Psychiatry. (2014) 85:1324-30. doi: 10.1136/jnnp-2013-306776

33. Biessels GJ, Koffeman A, Scheltens P. Diabetes and cognitive impairment Clinical diagnosis and brain imaging in patients attending a memory clinic. J Neurol. (2006) 253:477-82. doi: 10.1007/s00415-005-0036-4

34. Deary IJ, Corley J, Gow AJ, Harris SE, Houlihan LM, Marioni RE, et al. Age-associated cognitive decline. Br Med Bull. (2009) 92:135-52. doi: 10.1093/bmb/ldp033

35. Faraco G, Iadecola C. Hypertension: a harbinger of stroke and dementia. Hypertension. (2013) 62:810-7. doi: 10.1161/HYPERTENSIONAHA.113.01063

36. Knecht S, Oelschläger C, Duning T, Lohmann H, Albers J, Stehling C, et al. Atrial fibrillation in stroke-free patients is associated with memory impairment and hippocampal atrophy. Euro Heart J. (2008) 29:2125-32. doi: 10.1093/eurheartj/ehn341

37. Rafnsson SB, Deary IJ, Fowkes F. Peripheral arterial disease and cognitive function. Vasc Med. (2009) 14:51-61. doi: 10.1177/1358863x080 95027

38. McGovern A, Pendlebury ST, Mishra NK, Fan Y, Quinn TJ. Test accuracy of informant-based cognitive screening tests for diagnosis of dementia and multidomain cognitive impairment in stroke. Stroke. (2016) 47:329-35. doi: 10.1161/strokeaha.115.011218

39. Elias MF, Torres RV, Davey A. Clinical trials of blood pressure lowering and antihypertensive medication: is cognitive measurement state-of-the-art? Am J Hypertens. (2018). 31:631-42. doi: 10.1093/ajh/ hpy033

40. Hajduk AM, Kiefe CI, Person SD, Gore JG, Saczynski JS. Cognitive change in heart failure: a systematic review. Circ Cardiovasc Qual Outcomes. (2013) 6:451-60. doi: 10.1161/circoutcomes.113.000121

41. Alagiakrishnan K, McCracken P, Feldman H. Treating vascular risk factors and maintaining vascular health: is this the way towards successful cognitive ageing and preventing cognitive decline? Postgrad Med J. (2006) 82:101. doi: 10.1136/pgmj.2005.035030

Conflict of Interest: The authors declare that the research was conducted in the absence of any commercial or financial relationships that could be construed as a potential conflict of interest.

Copyright (C) 2020 Nicolas, Levi, Evans, Michie, Magin, Quain, Bivard and Karayanidis. This is an open-access article distributed under the terms of the Creative Commons Attribution License (CC BY). The use, distribution or reproduction in other forums is permitted, provided the original author(s) and the copyright owner(s) are credited and that the original publication in this journal is cited, in accordance with accepted academic practice. No use, distribution or reproduction is permitted which does not comply with these terms. 